\title{
GROWTH OF HARMONIC CONJUGATES IN THE UNIT DISC
}

\author{
MIROLJUB JEVTIĆ
}

Abstract. Assuming some mild regularity conditions on a positive nondecreasing function $\psi(x)=O\left(x^{a}\right)$ (for some $\left.a>0, x \rightarrow \infty\right)$, we show that

$$
M_{p}(r, u)=O\left(\psi\left(\frac{1}{1-r}\right)\right) \quad(r \rightarrow 1,0<p<1)
$$

implies $M_{p}(r, v)=O\left(\tilde{\psi}^{p}(1 /(1-r))\right)^{1 / p}$, where $u(z)+i v(z)$ is holomorphic in the open unit disc and

$$
\tilde{\psi}^{p}(x)=\int_{1 / 2}^{x} \frac{\psi^{p}(t)}{t} d t, \quad x \geqslant \frac{1}{2}
$$

1. Introduction. Throughout this note $\psi$ will denote a positive nondecreasing function defined for real $x \geqslant 0$. For each such function $\psi$ we define another function by

$$
\tilde{\psi}(x)=\int_{1 / 2}^{x} \frac{\psi(t)}{t} d t, \quad x \geqslant \frac{1}{2} .
$$

Throughout this paper $C$ denotes a positive constant, not necessarily the same at each occurrence.

A function $\varphi$ is almost increasing for $x \geqslant 0$ if there exists a positive constant $c$ such that $x_{1}<x_{2}$ implies $\varphi\left(x_{1}\right) \leqslant c \varphi\left(x_{2}\right)$. An almost decreasing function is defined similarly.

Let $u$ be a harmonic function in the open unit disc $U$ and, as usual, denote

$$
M_{p}(r, u)=\left(\frac{1}{2 \pi} \int_{0}^{2 \pi}\left|u\left(r e^{i t}\right)\right|^{p} d t\right)^{1 / p}, \quad 0<p<\infty,
$$

and

$$
M_{\infty}(r, u)=\sup \left\{\left|u\left(r e^{i t}\right)\right|, 0 \leqslant t \leqslant 2 \pi\right\} .
$$

Assuming that $\psi(x) / x^{a}$ is almost decreasing for some $a>0$, A. Shields and D. Williams in [4] showed that if $M_{\infty}(r, u)=O(\psi(1 /(1-r))), r \rightarrow 1$, then its conjugate $v$ satisfies $M_{\infty}(r, v)=O(\tilde{\psi}(1 /(1-r))), r \rightarrow 1$. They also showed that this

Received by the editors January 4, 1985.

1980 Mathematics Subject Classification (1985 Revision). Primary 31A05.

Key words and phrases. Conjugate functions. 
theorem remains valid if we replace $M_{\infty}(r, u)$ by $M_{1}(r, u)$. If $1<p<\infty$, the well-known theorem of M. Riesz [1, p. 54] says that $M_{p}(r, u)=O(\psi(1 /(1-r)))$ implies $M_{p}(r, v)=O(\psi(1 /(1-r)))$.

In this paper we shall be concerned only with means $M_{p}(r, u)$ when $0<p<1$. Hardy and Littlewood [3] proved that if $M_{p}(r, u)=O(1)$ for some $0<p<1$, then its conjugate $v$ satisfies $M_{p}(r, v)=O\left((\log 1 /(1-r))^{1 / p}\right)$. They also showed that if $M_{p}(r, u)=O\left((1-r)^{-\alpha}\right), \alpha>0,0<p<1$, then $M_{p}(r, v)$ satisfies the same growth condition. We fill the gap between these two results.

TheOREM. Let $u$ be harmonic in the unit disc. If there exists a $>0$ such that $\psi(x) / x^{a}$ is almost decreasing for $x \geqslant 1 / 2$ and if $M_{p}(r, u)=O(\psi(1 /(1-r)))$, for some $p, 0<p<1$, then the harmonic conjugate $v$ satisfies

$$
M_{p}(r, v)=O\left(\left(\tilde{\psi}^{p}(1 /(1-r))\right)^{1 / p}\right) .
$$

If $\psi(x)$ grows like $x^{\alpha}, \alpha>0$, then so does $\left(\tilde{\psi}^{p}\right)^{1 / p}$ and one obtains the theorem of Hardy and Littlewood. If $\psi(x) \equiv 1$, then $\left(\tilde{\psi}^{p}\right)^{1 / p}$ grows like $(\log x)^{1 / p}$, thus we recapture the bounded case mentioned above.

2. Proof of the theorem. We will need a lemma.

LEMMA. Let $\psi$ satisfy the conditions of the theorem. If $0<p<1$, then there exists $C>0$ such that, for all $x \geqslant 1$,

$$
(\tilde{\psi}(x))^{p} \leqslant C \tilde{\psi}^{p}(x) .
$$

Proof. Since $\psi$ is nondecreasing we have

$$
\tilde{\psi}(x)=\int_{1 / 2}^{x} \frac{\psi(t)}{t} d t \leqslant(\psi(x))^{1-p} \tilde{\psi}^{p}(x) .
$$

By Lemma 1(ii) of [4], $\tilde{\psi}$ grows faster then $\psi$; there exists $C>0$ such that, for all $x \geqslant 1, \psi(x) \leqslant C \tilde{\psi}(x)$. Hence,

$$
(\tilde{\psi}(x))^{p} \leqslant C \tilde{\psi}^{p}(x) .
$$

ProOF OF THE THEOREM. Without loss of generality we may suppose that $u$ is real and $u(0)=0$. Let $f(z)=u(z)+i v(z)=\sum_{n=1}^{\infty} \hat{f}(n) z^{n}$ be a holomorphic function on $U$. The fractional derivative of $f$ of first order is defined as

$$
f^{[1]}(z)=\sum_{n=1}^{\infty}(n+1) \hat{f}(n) z^{n}
$$

Note that

$$
f(z)=\int_{0}^{1} f^{[1]}(t z) d t .
$$


Let $r_{n}=1-2^{-n}$. Then

$$
\begin{aligned}
\left|f\left(r e^{i \theta}\right)\right|^{p} & =|f(z)|^{p}=\left|\int_{0}^{1} f^{[1]}(t z) d t\right|^{p} \\
& \leqslant\left(\sum_{n=1}^{\infty} \int_{r_{n-1}}^{r_{n}} \sup _{0 \leqslant t \leqslant \rho}\left|f^{[1]}(t z)\right| d \rho\right)^{p} \\
& \leqslant\left(\sum_{n=1}^{\infty} \sup _{0 \leqslant t \leqslant r_{n}}\left|f^{[1]}(t z)\right| 2^{-n}\right)^{p} \\
& \leqslant \sum_{n=1}^{\infty} 2^{-n p} \sup _{0 \leqslant t \leqslant r_{n}}\left|f^{[1]}(t z)\right|^{p} \\
& \leqslant C \sum_{n=1}^{\infty} \sup _{0 \leqslant t \leqslant r_{n}}\left|f^{[1]}(t z)\right|^{p} \int_{r_{n}}^{r_{n+1}}(1-\rho)^{p-1} d \rho \\
& \leqslant C \sum_{n=1}^{\infty} \int_{r_{n}}^{r_{n+1}} \sup _{0 \leqslant t \leqslant \rho}\left|f^{[1]}(t z)\right|^{p}(1-\rho)^{p-1} d \rho \\
& \leqslant C \int_{0}^{1}(1-\rho)^{p-1} \sup _{0 \leqslant t \leqslant \rho}\left|f^{[1]}(t z)\right|^{p} d \rho .
\end{aligned}
$$

If we now integrate on $\theta$ and use the Hardy-Littlewood maximal theorem [1, p. 12] we obtain

$$
M_{p}(r, f)^{p} \leqslant C \int_{0}^{1}(1-t)^{p-1} M_{p}\left(t r, f^{[1]}\right)^{p} d t .
$$

T. Flett [2, p. 762] proved that if $0<p<1$ and $1 / 3 \leqslant r<1$, then

$$
M_{p}\left(r, f^{\prime}\right)^{p} \leqslant C(1-r)^{-p-1} \int_{(3 r-1) / 2}^{(1+r) / 2} M_{p}(t, u)^{p} d t
$$

Thus,

(2)

$$
\begin{aligned}
M_{p}\left(r, f^{\prime}\right)^{p} & \leqslant C(1-r)^{-p-1} \int_{(3 r-1) / 2}^{(1+r) / 2} \psi^{p}\left(\frac{1}{1-t}\right) d t \\
& =C(1-r)^{-p-1} \int_{2(1-r)^{-1} / 3}^{2(1-r)^{-1}}[\psi(t)]^{p} \cdot t^{-2} d t \\
& =C(1-r)^{-p-1} \int_{2(1-r)^{-1} / 3}^{2(1-r)^{-1}}\left[\psi(t) / t^{a}\right]^{p} t^{a p-2} d t \\
& \leqslant C(1-r)^{a p-p-1}\left[\psi\left(\frac{1}{1-r}\right)\right]^{p} \int_{2(1-r)^{-1} / 3}^{2(1-r)^{-1}} t^{a p-2} d t \\
& \leqslant C(1-r)^{-p} \psi^{p}\left(\frac{1}{1-r}\right)
\end{aligned}
$$

The inequality

$$
M_{p}\left(r, f^{[1]}\right) \leqslant C M_{p}\left(r, f^{\prime}\right)
$$

is obvious since $f^{[1]}(z)=f(z)+z f^{\prime}(z)$. 
Combining (1), (2) and (3) we obtain

$$
M_{p}(r, f)^{p} \leqslant C \int_{0}^{1}(1-t)^{p-1}(1-t r)^{-p} \psi^{p}\left(\frac{1}{1-t r}\right) d t .
$$

By Lemma 1(iii) of [4],

$$
\psi^{p}\left(\frac{1}{1-r}\right) \leqslant C(1-r) \sum_{n=0}^{\infty} \psi^{p}(n) r^{n} .
$$

Hence, from (4), it follows that

$$
\begin{aligned}
M_{p}(r, f)^{p} \leqslant & C \int_{0}^{1}\left(\frac{1-r t}{1-t}\right)^{1-p}\left(\sum_{n=0}^{\infty} \psi^{p}(n) r^{n} t^{n}\right) d t \\
\leqslant & C\left(\sum_{n=0}^{\infty} \psi^{p}(n) r^{n}\left(\int_{0}^{r}\left(\frac{1-r t}{1-t}\right)^{1-p} t^{n} d t\right)\right. \\
& \left.+\sum_{n=0}^{\infty} \psi^{p}(n) r^{n}\left(\int_{r}^{1}\left(\frac{1-r t}{1-t}\right)^{1-p} t^{n} d t\right)\right) .
\end{aligned}
$$

We now show that each term in (5) is $O\left(\tilde{\psi}^{p}(1 /(1-r))\right)$. Applying Lemma $1(v)$ of [4] to the function $\psi^{p}(x)$ we have

$$
\sum_{n=0}^{\infty} \frac{\psi^{p}(n)}{n+1} r^{n} \leqslant C \tilde{\psi}^{p}\left(\frac{1}{1-r}\right) .
$$

Hence,

$$
\begin{aligned}
\sum_{n=0}^{\infty} \psi^{p}(n) r^{n}\left(\int_{0}^{r}\left(\frac{1-r t}{1-t}\right)^{1-p} t^{n} d t\right) & \leqslant C \sum_{n=0}^{\infty} \psi^{p}(n)\left(\int_{0}^{1} t^{n} d t\right) r^{n} \\
& \leqslant C \sum_{n=0}^{\infty} \frac{\psi^{p}(n)}{n+1} r^{n} \leqslant C \tilde{\psi}^{p}\left(\frac{1}{1-r}\right) .
\end{aligned}
$$

It is a simple consequence of Jensen's inequality that

$$
(1-r) \sum_{n=0}^{\infty}\left[\frac{\psi(n)}{n+1}\right]^{p} r^{n} \leqslant\left\{(1-r) \cdot \sum_{n=0}^{\infty} \frac{\psi(n)}{n+1} r^{n}\right\}^{p} .
$$

For the second term, we have

$$
\begin{aligned}
\sum_{n=0}^{\infty} \psi^{p}(n) r^{n} & \left(\int_{r}^{1}\left(\frac{1-r t}{1-t}\right)^{1-p} t^{n} d t\right) \\
& \leqslant C(1-r)^{1-p} \sum_{n=0}^{\infty} \psi^{p}(n)\left(\int_{0}^{1}(1-t)^{p-1} t^{n} d t\right) r^{n} \\
& \leqslant C(1-r)^{1-p} \sum_{n=0}^{\infty} \psi^{p}(n) \frac{\Gamma(p) \Gamma(n+1)}{\Gamma(n+p+1)} r^{n} \\
& \leqslant C(1-r)^{1-p} \sum_{n=0}^{\infty} \frac{\psi^{p}(n)}{(n+1)^{p}} r^{n} .
\end{aligned}
$$


From (6), Lemma 1(v) of [4], and the lemma it follows that the second term on the right-hand side of $(5)$ is also $O\left(\tilde{\psi}^{p}(1 /(1-r))\right)$.

3. Remarks. A function $\psi$ is normal if there exist $a, b>0$ such that $\psi(x) / x^{a}$ is almost decreasing for $x \geqslant 1 / 2$ and $\psi(x) / x^{b}$ is almost increasing for $x \geqslant 1 / 2$. If $0<p<1$ and $\psi$ is normal, then $\psi, \tilde{\psi}$ and $\left(\tilde{\psi}^{p}\right)^{1 / p}$ have the same rate of growth. Thus, when $\psi$ is normal the theorem says that if $u$ is harmonic in the unit disc and $M_{p}(r, u)=O(\psi(1 /(1-r)))$, then $M_{p}(r, v)=O(\psi(1 /(1-r)))$. If $\psi$ satisfies the conditions of the theorem, but is not normal, then $\left(\tilde{\psi}^{p}\right)^{1 / p}$ grows at a faster rate than $\psi$. I do not know whether the theorem is best possible, i.e., is there a harmonic function $u$ such that

$$
M_{p}(r, u)=O\left(\psi\left(\frac{1}{1-r}\right)\right) \text { and } M_{p}(r, v) \geqslant C\left(\tilde{\psi}^{p}\left(\frac{1}{1-r}\right)\right)^{1 / p} ?
$$

We note that Hardy and Littlewood proved in [3] that if $k$ is a positive integer, $p=1 /(k+1)$ and $f(z)=\exp \left(\frac{1}{2} k \pi i\right)(1-z)^{-k-1}$ then $M_{p}(r, \operatorname{Re} f)$ is bounded and $M_{p}(r, f) \sim(\log (1 /(1-r)))^{1 / p}, r \rightarrow 1$.

\section{REFERENCES}

1. P. L. Duren, Theory of $H^{p}$ spaces, Academic Press, New York, 1970.

2. T. M. Flett, The dual of an inequality of Hardy and Littlewood and some related inequalities, J. Math. Anal. Appl. 38 (1972), 746-765.

3. G. H. Hardy and J. E. Littlewood, Some properties of conjugate functions, J. Reine Angew. Math. 167 (1931), 405-423.

4. A. L. Shields and D. L. Williams, Bounded projections and the growth of harmonic conjugates in the unit disc, Michigan Math. J. 29 (1982), 3-25.

InSTItUt Za Mathematiku, StUdentski TRg 16, 11000 Beograd, Yugoslavia 